\title{
Lace expansion for dummies
}

\author{
Erwin Bolthausen ${ }^{\mathrm{a}, 1}$, Remco van der Hofstad ${ }^{\mathrm{b}, 2}$ and Gady Kozma ${ }^{\mathrm{c}, 3}$ \\ anstitut für Mathematik, Universität Zürich, Winterthurerstrasse 190, 8057 Zürich, Switzerland and Research Institute for Mathematical \\ Sciences, Kyoto University, Kyoto 606-8502, Japan.E-mail: eb@math.uzh.ch \\ ${ }^{\mathrm{b}}$ Department of Mathematics and Computer Science, Eindhoven University of Technology, P.O. Box 513, 5600 MB Eindhoven, The Netherlands. \\ E-mail: r.w.v.d.hofstad@tue.nl \\ ${ }^{\mathrm{c}}$ Weizmann Institute, Rehovot, 76100, Israel. E-mail: gady.kozma@weizmann.ac.il
}

Received 26 April 2016; revised 27 August 2016; accepted 22 September 2016

\begin{abstract}
We show Green's function asymptotic upper bound for the two-point function of weakly self-avoiding walk in $d>4$, revisiting a classic problem. Our proof relies on Banach algebras to analyse the lace-expansion fixed point equation and is simpler than previous approaches in that it avoids Fourier transforms.
\end{abstract}

Résumé. Nous montrons une domination asymptotique de la fonction à deux points de la marche faiblement auto-évitante en dimension $d>4$ par la fonction de Green, revisitant ainsi un problème classique. Notre preuve s'appuie sur des techniques d'algèbres de Banach pour analyser le point fixe de l'équation de développement en lacets. Elle est plus simple que les approches précédentes car elle ne passe pas par la transformée de Fourier.

MSC: 84B41; 60K35

Keywords: Self-avoiding walk; Lace expansion; Banach algebra; Deconvolution; Edgeworth expansion

\section{Introduction}

The lace expansion made its debut in 1985 with a proof by Brydges and Spencer that weakly self-avoiding walk (WSAW) has "Gaussian behaviour" in dimensions 5 and above [4]. It proved to be useful way beyond its initial application, primarily in work by Hara and Slade. The technique was applied to percolation [7], lattice trees and animals [8], the contact process [12], the Ising model [13] and $\varphi^{4}$ [14]. Further, it was extended to finite graphs [3] and to long-range models [5]. Despite all this progress, weakly self-avoiding walk remains the simplest example to which the technique applies: lace expansion is a "perturbative" technique and it requires a small parameter. Weakly self-avoiding walk has such a small parameter naturally built-in, while for most models, the small parameter is more hidden. Consequently, it was used as a test bed for several new techniques, for example in [10], where the lace expansion was analysed using induction in time, and [2] where a Banach fixed point theorem was used. Interestingly, neither of these papers uses the so-called bootstrap analysis introduced in [15]. In our opinion, the bootstrap analysis is the most important simplification to lace expansion, replacing the difficult "moving single pole" analysis of [4]. The bootstrap analysis applies to generating functions such as the WSAW Green's function, while $[2,10]$ prove results for WSAW with a fixed number of steps instead. Green's function asymptotics in $x$-space as derived here were proved

\footnotetext{
${ }^{1}$ Supported by SNF Grant 200020_138141.

${ }^{2}$ Supported by the Netherlands Organisation for Scientific Research (NWO) through VICI Grant 639.033.806 and the Gravitation NETwORKS Grant 024.002.003.

${ }^{3}$ Supported by the Israel Science Foundation and the Jesselson Foundation, and by the CNRS during his visit to the Institut Heni Poincaré.
} 
previously in technically more challenging settings in [9] for spread-out models and in [6] for nearest-neighbour settings. Brydges and Spencer [4] prove Gaussian limit laws for the end-to-end displacement for WSAW after $n$ steps.

Our starting point was also an attempt to generalise lace expansion, rather than to simplify it. We wished to apply it to problems on Cayley graphs of non-commutative groups. Most of the existing approaches rely heavily on the Fourier transform, which is of course no longer available in this new setting. The approach of [1,2], though, turned out to be applicable. We realised that it can be simplified and generalised by working in an appropriate Banach algebra.

In this paper, we expose our Banach-algebra approach in the simplest possible setting: weakly self-avoiding walk on $\mathbb{Z}^{d}$, with the result being an upper bound on the critical Green's function. We repeat that related results have been proved previously, only our proof is novel.

\section{Precise definitions and statement of the theorem}

For a nearest-neighbour path $\gamma:\{0, \ldots, n\} \rightarrow \mathbb{Z}^{d}$ and a $\beta \in[0,1]$, we define its weight by

$$
W(\gamma)=W^{\beta}(\gamma)=(1-\beta)^{|\{0 \leq s<t \leq n: \gamma(s)=\gamma(t)\}|},
$$

i.e., the path is "penalised" by $1-\beta$ for every self-intersection of $\gamma$. We define the weakly self-avoiding walk Green's function to be

$$
G_{\lambda}^{\text {saw }}(x)=G_{\lambda}^{\beta, \text { saw }}(x)=\sum_{\gamma: 0 \rightarrow x} \lambda^{\text {len }(\gamma)} W^{\beta}(\gamma),
$$

where the notation $\gamma: 0 \rightarrow x$ means that $\gamma:\{0,1, \ldots, n\} \rightarrow \mathbb{Z}^{d}$ is a nearest-neighbour path with $\gamma(0)=0, \gamma(n)=x$, while $\operatorname{len}(\gamma)=n$. We define $\lambda_{c}$ to be the critical value for the finiteness of the spatial sum of $G_{\lambda}^{\text {saw }}$, i.e.,

$$
\lambda_{c}=\sup \left\{\lambda: \sum_{x \in \mathbb{Z}^{d}} G_{\lambda}^{\mathrm{saw}}(x)<\infty\right\} .
$$

Finally denote by $G^{\mathrm{rw}}(x)$ the (critical) Green's function of simple random walk (SRW) on $\mathbb{Z}^{d}$, i.e.,

$$
G^{\mathrm{rw}}(x)=\sum_{n=0}^{\infty} p_{n}(x),
$$

where $p_{n}(x)$ is the probability that simple random walk on $\mathbb{Z}^{d}$ starting from 0 is at $x$ at time $n$. When $d>2$ the sum converges and

$$
G^{\mathrm{rw}}(x)=(a+o(1))|x|^{2-d} \quad \text { as }|x| \rightarrow \infty
$$

with $a>0$. See e.g. [17]. The result is that the WSAW Green's function is bounded by the SRW Green's function for $d>4$ :

Theorem (Green's function upper bound). Let $d>4$. Then there exists a $\beta_{0}$ such that for all $\beta<\beta_{0}, \beta$-weakly self avoiding walk satisfies

$$
G_{\lambda_{c}}^{\mathrm{saw}}(x) \leq 2 G^{\mathrm{rw}}(x) \quad \forall x \in \mathbb{Z}^{d} .
$$

Remark. We will also show a lower bound, $G_{\lambda_{c}}^{\text {saw }} \geq \frac{1}{2} G^{\text {rw }}$, and further that

$$
G_{\lambda_{c}}^{\text {saw }}(x)=(1+O(\beta)) G^{\mathrm{rw}}(x) \text { as } \beta \rightarrow 0 \text {, uniformly in } x \in \mathbb{Z}^{d} .
$$

See the remarks on page 152 . We find the upper bound to be the more interesting and we prefer to focus on it.

The remainder of this paper is devoted to the proof of this theorem. 


\section{Proof}

For $\mu \in \mathbb{R}$ we denote by $\Delta_{\mu}^{\mathrm{rw}}: \mathbb{Z}^{d} \rightarrow \mathbb{R}$ the following function

$$
\Delta_{\mu}^{\mathrm{rw}}(x)= \begin{cases}1, & x=0, \\ -\mu, & x \text { is a neighbour of } 0 \\ 0, & \text { otherwise. }\end{cases}
$$

We say that a function $f: \mathbb{Z}^{d} \rightarrow \mathbb{R}$ is "symmetric to coordinate permutations and flipping" if for any $\sigma \in S_{d}$ (the group of permutations on $d$ elements) and for any $\varepsilon_{1}, \ldots, \varepsilon_{d} \in\{ \pm 1\}$,

$$
f\left(x_{1}, \ldots, x_{d}\right)=f\left(\varepsilon_{1} x_{\sigma(1)}, \ldots, \varepsilon_{d} x_{\sigma(d)}\right) .
$$

We further write $f * g$ for the convolution of two functions $f, g: \mathbb{Z}^{d} \rightarrow \mathbb{R}$ and $\delta_{0}(x)=1_{\{x=0\}}, x \in \mathbb{Z}^{d}$.

Lemma 1 (Lace expansion analysis). Let $d>4$. Then there exists a $\beta_{0}$ such that for all $\beta<\beta_{0}$ and for all $\lambda<\lambda_{c}$ the following holds. If $G_{\lambda}^{\mathrm{saw}}(x) \leq 3 G^{\mathrm{rw}}(x)$ for all $x \in \mathbb{Z}^{d}$, then there exists a function $\Delta_{\lambda}^{\mathrm{saw}}: \mathbb{Z}^{d} \rightarrow \mathbb{R}$ such that $G_{\lambda}^{\mathrm{saw}} * \Delta_{\lambda}^{\mathrm{saw}}=\delta_{0}$ and such that

(i) $\Delta_{\lambda}^{\text {saw }}$ is symmetric to coordinate permutations and flipping;

(ii) $\sum_{x} \Delta_{\lambda}^{\text {saw }}(x) \geq 0$;

(iii) There exists $\lambda^{\prime} \in\left[0, \frac{1}{2 d}\right]$ such that

$$
\left|\Delta_{\lambda}^{\mathrm{saw}}(x)-\Delta_{\lambda^{\prime}}^{\mathrm{rw}}(x)\right| \leq C \beta|x|^{-d-4}
$$

A somewhat abusive convention we adopt here and below is that $|x|^{-\alpha}=1$ when $x=0$, so condition (iii) in fact implies that $\left|\Delta_{\lambda}^{\text {saw }}(0)-\Delta_{\lambda^{\prime}}^{\text {rw }}(0)\right| \leq C \beta$. $C$ and $c$ are used for constants that depend only on the dimension. Let us remark that in fact we simply take $\lambda^{\prime}=\min \left(\lambda, \frac{1}{2 d}\right)$, though we will not use this fact. Another remark worth making is that in (iii) we will in fact prove, $\left|\Delta_{\lambda}^{\text {saw }}(x)-\Delta_{\lambda^{\prime}}^{\mathrm{rw}}(x)\right| \leq C \beta|x|^{6-3 d}$ which is of course stronger than the stated estimate when $d \geq 5$. However, it will be convenient to formulate the lemma as above.

We remark that it is tempting to think about $\Delta_{\lambda}^{\text {saw }}$ as a generator of some random walk (with killing), but it is missing one important property of a generator: it is not true that $\Delta_{\lambda}^{\text {saw }}(x)<0$ for all $x \neq 0$. This means that a lot of deconvolution techniques for random walk generators are inapplicable. The next lemma is the required deconvolution:

Lemma 2 (Deconvolution). Let $d>2$. Then there exists $\beta_{0}$ such that for all $\beta<\beta_{0}$ and for any $\Delta: \mathbb{Z}^{d} \rightarrow \mathbb{R}$ satisfying conditions (i)-(iii) of Lemma 1 , there exists a function $G$ such that $G * \Delta=\delta_{0}$ and $|G(x)| \leq 2 G^{\mathrm{rw}}(x)$.

We postpone the proof of both lemmas and first show how they imply the theorem:

Proof of the theorem given Lemmas 1 and 2. Fix $\beta$ to be some value sufficiently small so that both Lemmas 1 and 2 hold with this value of $\beta$. The following argument, known as a bootstrap argument, goes back to Slade [15]. Define

$$
f(\lambda)=\sup _{x \in \mathbb{Z}^{d}} \frac{G_{\lambda}^{\mathrm{saw}}(x)}{G^{\mathrm{rw}}(x)} .
$$

We first examine $f(0) . G_{0}^{\text {saw }}=\delta_{0}$ and of course $G^{\mathrm{rw}} \geq \delta_{0}$ so $f(0) \leq 1$. Next we note that $f$ is continuous in the interval $\left[0, \lambda_{c}\right)$. Indeed, $\lambda_{c}$ is the radius of convergence of $\sum_{x} G_{\lambda}^{\text {saw }}(x)$ and hence is a lower bound for the radius of convergence of $G_{\lambda}^{\text {saw }}(x)$ for any $x$. Hence each term $G_{\lambda}^{\text {saw }}(x) / G^{\text {rw }}(x)$ is continuous on our interval. On the other hand, because the sum defining $G_{\lambda}^{\text {saw }}(x)$ contains only paths of length at least $|x|$, it also decays exponentially in $x$, uniformly on $[0, \lambda]$, for all $\lambda<\lambda_{c}$. Using (2), the same is true for the quotient $G_{\lambda}^{\mathrm{saw}}(x) / G^{\mathrm{rw}}(x)$. This means that on any $[0, \lambda]$ with $\lambda<\lambda_{c}, f$ can be written as the supremum of a finite collection of continuous functions, and hence is continuous. Since $\lambda$ can be taken arbitrarily close to $\lambda_{c}, f$ is continuous on $\left[0, \lambda_{c}\right)$. 
We now claim that it is not possible that $f(\lambda) \in(2,3]$ for any $\lambda<\lambda_{c}$. Indeed, if $f(\lambda) \leq 3$ then $G_{\lambda}^{\mathrm{saw}}(x) \leq 3 G^{\mathrm{rw}}(x)$ for all $x$ and the condition of Lemma 1 is satisfied. We use Lemma 1 to find some $\Delta_{\lambda}^{\text {saw }}$ with $G_{\lambda}^{\text {saw }} * \Delta_{\lambda}^{\text {saw }}=\delta_{0}$ satisfying conditions (i)-(iii), and then Lemma 2 to find some $G$ such that $G * \Delta_{\lambda}^{\text {saw }}=\delta_{0}$ and $G(x) \leq 2 G^{\mathrm{rw}}(x)$. We now claim that $G_{\lambda}^{\text {saw }}=G$. Indeed, both functions are in $\ell^{2}\left(\mathbb{Z}^{d}\right), G_{\lambda}^{\text {saw }}$ by the assumption $G_{\lambda}^{\text {saw }}(x) \leq 3 G^{\text {rw }}(x)$ and $G$ by the conclusion of Lemma 2 - and so is $\Delta_{\lambda}^{\text {saw }}$ by condition (iii). In $\ell^{2}$, deconvolution can be performed by Fourier transform and hence is unique. We get that $G_{\lambda}^{\text {saw }}(x)=G(x) \leq 2 G^{\mathrm{rw}}(x)$ so $f(\lambda) \leq 2$. We conclude that $f(\lambda) \notin(2,3]$ for any $\lambda<\lambda_{c}$.

Now, if $f$ is continuous, starts below 1 and cannot traverse the interval $(2,3]$, then it must be that $f(\lambda) \leq 2$ for all $\lambda<\lambda_{c}$, i.e., $G_{\lambda}^{\text {saw }}(x) \leq 2 G^{\mathrm{rw}}(x)$ for all $x$ and all $\lambda<\lambda_{c}$. Finally, by monotone convergence, $G_{\lambda_{c}}^{\text {saw }}(x)=$ $\lim _{\lambda \succ \lambda_{c}} G_{\lambda}^{\text {saw }}(x)$, so that also $G_{\lambda_{c}}^{\text {saw }}(x) \leq 2 G^{\mathrm{rw}}(x)$ for all $x \in \mathbb{Z}^{d}$.

We move to the proofs of Lemmas 1 and 2. Lemma 1 essentially relies on the same lace-expansion argument as performed by Brydges and Spencer [4] - we include the proof for completeness, but we will be a little brief. Lemma 2 is the new ingredient of our paper.

Proof of Lemma 1. We follow [10, Appendix A] closely for the derivation of the lace expansion, and [9] for the analysis of the coefficients arising in it. As $\beta$ and $\lambda$ are fixed, let us remove them from the notation and denote our functions by $G^{\text {saw }}$ and $\Delta^{\text {saw }}$. We start by finding a formula for $\Delta^{\text {saw }}$ (or rather, a representation as an infinite sum). Recall the weight $W(\gamma)$ defined in (1). We define

$$
U_{s t}(\gamma)=\left\{\begin{array}{ll}
0, & \text { when } \gamma(s) \neq \gamma(t), \\
-\beta, & \text { when } \gamma(s)=\gamma(t),
\end{array} \quad \forall 0 \leq s<t \leq \operatorname{len}(\gamma),\right.
$$

so that

$$
W(\gamma)=\prod_{s<t}\left(1+U_{s t}(\gamma)\right) .
$$

Given an interval $I=[a, b]$ of integers with $0 \leq a \leq b$, we refer to a pair $\{s, t\}(s<t)$ of elements of $I$ as an edge. To abbreviate the notation, we write $s t$ for $\{s, t\}$. A set of edges is called a graph. A graph $\Gamma$ on $[a, b]$ is said to be connected if both $a$ and $b$ are endpoints of edges in $\Gamma$ and if, in addition, for any $c \in(a, b)$ there is an edge $s t \in \Gamma$ such that $s<c<t$ (note that this is unrelated to the usual definition of graph connectivity). The set of all graphs on $[a, b]$ is denoted $\mathscr{B}[a, b]$, and the subset consisting of all connected graphs is denoted $\mathscr{G}[a, b]$.

For integers $0 \leq a<b$, define

$$
K[a, b](\gamma)=\prod_{a \leq s<t \leq b}\left(1+U_{s t}(\gamma)\right) \quad \text { so that } G^{\mathrm{saw}}(x)=\sum_{\gamma: 0 \rightarrow x} \lambda^{\operatorname{len}(\gamma)} K[0, \operatorname{len}(\gamma)](\gamma),
$$

where the sum is over all simple random walk paths from 0 to $x$. Expanding the product in the definition of $K[a, b](\gamma)$, we get

$$
K[a, b](\gamma)=\sum_{\Gamma \in \mathscr{B}[a, b]} \prod_{s t \in \Gamma} U_{s t}(\gamma) .
$$

For $0 \leq a<b$ we define an analogous quantity, in which the sum over graphs is restricted to connected graphs, namely,

$$
J[a, b](\gamma)=\sum_{\Gamma \in \mathscr{G}[a, b]} \prod_{s t \in \Gamma} U_{s t}(\gamma)
$$

We claim that, for $n \geq 1$,

$$
K[0, n]=K[1, n]+\sum_{m=2}^{n} J[0, m] K[m, n] .
$$


To see this, we note from (6) that the contribution to $K[0, n]$ from all graphs $\Gamma$ for which 0 is not in an edge is exactly $K[1, n]$. To resum the contribution from the remaining graphs, we proceed as follows. When $\Gamma$ does contain an edge ending at 0 , we let $m(\Gamma)>0$ denote the smallest number that is not crossed by an edge, i.e., there is no $s t \in \Gamma$ such that $s<m(\Gamma)<t$. We lose nothing by taking $m \geq 2$, since $U_{a, a+1}=0$ for all $a$. Resummation over graphs on $[m, n]$ and (7) proves (8).

Let us now define the key quantities in the lace expansion, which are

$$
\Pi_{\lambda}(x)=\sum_{\gamma: 0 \rightarrow x} \lambda^{\operatorname{len}(\gamma)} J[0, \operatorname{len}(\gamma)](\gamma)
$$

and

$$
\Delta_{\lambda}^{\mathrm{saw}}(x):=\Delta_{\lambda}^{\mathrm{rw}}(x)-\Pi_{\lambda}(x) .
$$

$\lambda$ will be fixed through the rest of the proof of Lemma 1 and we usually drop it from the notation. The key to the proof of the lemma is the estimate

$$
\sum_{\substack{n=0 \\ \text { len } \gamma=n}}^{\infty} \sum_{\substack{\gamma: 0 \rightarrow x \\ \text { la }}}\left|\lambda^{n} J[0, n](\gamma)\right|<C \beta|x|^{6-3 d},
$$

which we will prove below. We first prove that it implies the claims in the lemma. Of course it implies

$$
|\Pi(x)| \leq C \beta|x|^{6-3 d} .
$$

To conclude from the definitions and estimate above that $G^{\mathrm{saw}} * \Delta^{\mathrm{saw}}=\delta_{0}$ note that, by (8),

$$
\begin{aligned}
G^{\mathrm{saw}}(x) & \stackrel{(5)}{=} \sum_{\gamma: 0 \rightarrow x} \lambda^{\operatorname{len}(\gamma)} K[0, \operatorname{len}(\gamma)](\gamma) \\
& \stackrel{(8)}{=} \delta_{0}+\sum_{\gamma: 0 \rightarrow x} \lambda^{\operatorname{len}(\gamma)}\left(K[1, \operatorname{len}(\gamma)](\gamma)+\sum_{m=2}^{\operatorname{len}(\gamma)} J[0, m](\gamma) K[m, \operatorname{len}(\gamma)](\gamma)\right) \\
& =\delta_{0}+\lambda \sum_{y:\|y\|=1} G^{\mathrm{saw}}(x-y)+\sum_{y} \Pi(y) G^{\mathrm{saw}}(x-y),
\end{aligned}
$$

where the last equality is derived as follows: the $K[1, \operatorname{len}(\gamma)](\gamma)$ terms we separate according to $\gamma(1)$, which we denote by $y$. Translation invariance gives that each term is exactly $G^{\text {saw }}(x-y)$. The terms containing $J$ are split according to $\gamma(m)$, which we denote by $y$, and again by translation invariance the sum over $K$ gives $G^{\text {saw }}(x-y)$. Finally, the change of order of summation is justified by (11) and $G^{\text {saw }}(x) \leq C|x|^{2-d}$. This proves (13). Rearranging (13) gives $G^{\text {saw }} * \Delta_{\lambda}^{\text {rw }}=\delta_{0}+G^{\text {saw }} * \Pi$, i.e. $G^{\text {saw }} * \Delta_{\lambda}^{\text {saw }}=\delta_{0}$, as required.

We move to prove properties (i)-(iii) of $\Delta^{\text {saw }}$. The symmetry of $\Delta^{\text {saw }}$ is immediate from the construction, and the property that $\sum \Delta^{\text {saw }}(x) \geq 0$ comes from summing the relation $\left(G^{\text {saw }} * \Delta^{\text {saw }}\right)(x)=\delta_{0}(x)$ over $x \in \mathbb{Z}^{d}$, which gives that

$$
\sum_{x \in \mathbb{Z}^{d}} \Delta^{\mathrm{saw}}(x)=\frac{1}{\sum_{x \in \mathbb{Z}^{d}} G^{\mathrm{saw}}(x)},
$$

and the last term is clearly non-negative as well as finite since $\lambda<\lambda_{c}$ which means that $G^{\text {saw }}(x)$ decays exponentially as $x \rightarrow \infty$.

For (iii), we need to choose $\lambda^{\prime} \in\left(0, \frac{1}{2 d}\right]$ and estimate $\Delta_{\lambda}^{\mathrm{saw}}-\Delta_{\lambda^{\prime}}^{\mathrm{rw}} \stackrel{(10)}{=} \Delta_{\lambda}^{\mathrm{rw}}-\Delta_{\lambda^{\prime}}^{\mathrm{rw}}-\Pi_{\lambda}$. Observe first that by (12), we can take $\lambda^{\prime}=\lambda$ if $\lambda \leq 1 / 2 d$. If $\lambda>1 / 2 d$, we obtain from (12) and the already proved (ii)

$$
1-2 d \lambda=\sum_{x} \Delta_{\lambda}^{\mathrm{rw}}(x) \stackrel{(10)}{=} \sum_{x}\left(\Pi_{\lambda}(x)+\Delta_{\lambda}^{\mathrm{saw}}(x)\right) \stackrel{(\mathrm{ii})}{\geq} \sum_{x} \Pi_{\lambda}(x) \stackrel{(12)}{\geq}-\sum_{x} C \beta|x|^{6-3 d}=-C \beta,
$$




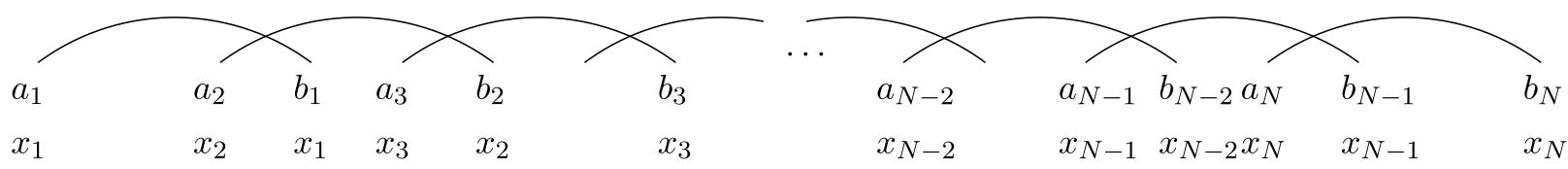

Fig. 1. Laces.

and therefore $\lambda-1 / 2 d \leq C \beta / 2 d$, and so we can take $\lambda^{\prime}=1 / 2 d$ in this case. Therefore, $\lambda^{\prime}=\min \left(\lambda, \frac{1}{2 d}\right)$ is doing the job.

It remains to prove (11).

We next rewrite (9) in a form that can be used to obtain good bounds on $\Pi(x)$. For this, we start by introducing the laces that give the lace expansion its name. A lace is a minimally connected graph, i.e., a connected graph for which the removal of any edge would result in a disconnected graph. The set of laces on $[a, b]$ is denoted $\mathscr{L}[a, b]$, and the set of laces on $[a, b]$ consisting of exactly $N$ edges is denoted $\mathscr{L}^{(N)}[a, b]$. Given a connected graph $\Gamma$, the following prescription associates to $\Gamma$ a unique lace $L_{\Gamma}$ : The lace $L_{\Gamma}$ consists of edges $a_{1} b_{1}, a_{2} b_{2}, \ldots$, with $a_{1}, b_{1}, b_{2}, a_{2}, \ldots$ determined, in that order, by

$$
\begin{aligned}
& b_{1}=\max \{t: a t \in \Gamma\}, \quad a_{1}=a, \\
& b_{i}=\max \left\{t: \exists z<b_{i-1} \text { such that } z t \in \Gamma\right\}, \quad a_{i}=\min \left\{z: z b_{i} \in \Gamma\right\} .
\end{aligned}
$$

See Figure 1. Given a lace $L$, the set of all edges $s t \notin L$ such that $L_{L \cup\{s t\}}=L$ is denoted $\mathscr{C}(L)$. Edges in $\mathscr{C}(L)$ are said to be compatible with $L$. Now, $L_{\Gamma}=L$ if and only if $L \subset \Gamma$ and all edges in $\Gamma \backslash L$ are compatible with $L$. This allows to write

$$
\sum_{\Gamma: L_{\Gamma}=L} \prod_{s t \in \Gamma \backslash L} U_{s t}=\prod_{s t \in \mathscr{C}(L)}\left(1+U_{s t}\right)
$$

and then partially resum the right-hand side of (7), to obtain

$$
J[a, b]=\sum_{L \in \mathscr{L}[a, b]} \sum_{\Gamma: L_{\Gamma}=L} \prod_{s t \in L} U_{s t} \prod_{s^{\prime} t^{\prime} \in \Gamma \backslash L} U_{s^{\prime} t^{\prime}}=\sum_{L \in \mathscr{L}[a, b]} \prod_{s t \in L} U_{s t} \prod_{s^{\prime} t^{\prime} \in \mathscr{C}(L)}\left(1+U_{s^{\prime} t^{\prime}}\right) .
$$

For $0 \leq a<b$, we define $J^{(N)}[a, b]$ to be the contribution to (15) coming from laces consisting of exactly $N$ edges:

$$
J^{(N)}[a, b]=\sum_{L \in \mathscr{L}^{(N)}[a, b]} \prod_{s t \in L}\left(-U_{s t}\right) \prod_{s^{\prime} t^{\prime} \in \mathscr{C}(L)}\left(1+U_{s^{\prime} t^{\prime}}\right), \quad N \geq 1 .
$$

Then, by (9),

$$
J[a, b]=\sum_{N=1}^{\infty}(-1)^{N} J^{(N)}[a, b] \text { and } \Pi(x)=\sum_{N=1}^{\infty}(-1)^{N} \Pi^{(N)}(x),
$$

where we define

$$
\begin{aligned}
\Pi^{(N)}(x) & =\sum_{\gamma: 0 \rightarrow x} \lambda^{\operatorname{len}(\gamma)} J^{(N)}[0, \operatorname{len}(\gamma)](\gamma) \\
& =\sum_{\gamma: 0 \rightarrow x} \lambda^{\operatorname{len}(\gamma)} \sum_{L \in \mathscr{L}^{(N)}[0, \operatorname{len}(\gamma)]} \prod_{s t \in L}\left(-U_{s t}(\gamma)\right) \prod_{s^{\prime} t^{\prime} \in \mathscr{C}(L)}\left(1+U_{s^{\prime} t^{\prime}}(\gamma)\right) .
\end{aligned}
$$

We will now show that the sum over $N$ converges absolutely, which will show (11) and finish the lemma. The product over $s t \in \mathscr{C}(L)$ will be easier to handle when we restrict it. Let therefore $\mathscr{D}(L)$ be the set of edges st such that the open interval $(s, t)$ does not contain an $a_{i}$ or $b_{i}$ for any $\left(a_{i}, b_{i}\right) \in L$. Clearly $\mathscr{D}(L) \subseteq \mathscr{C}(L)$ and therefore 
$\prod_{\mathscr{C}(L)}\left(1+U_{s t}\right) \leq \prod_{\mathscr{D}(L)}\left(1+U_{s t}\right)$. Once we restrict, the sum over $\gamma$ becomes independent between any two consecutive elements of $L$. For a lace $L=\left\{a_{1} b_{1}, a_{2} b_{2}, \ldots, a_{N} b_{N}\right\}$ we write $c_{1}, c_{2}, \ldots, c_{2 N}$ for the ordered sequence of vertices of the bonds of $L$, i.e. $c_{1}=a_{1}, c_{2}=a_{2}, c_{3}=b_{1}, \ldots$. There can be matches among the $c$ 's, e.g. for $N=3, b_{1}$ can be equal to $a_{3}$. We denote by $\gamma_{i}, 1 \leq i \leq 2 N-1$, the $\gamma$-path restricted to the interval $\left\{c_{i}, c_{i}+1, \ldots, c_{i+1}\right\}$, and we get, for $N \geq 2$,

$$
\prod_{s t \in \mathscr{D}(L)}\left(1+U_{s t}(\gamma)\right)=\prod_{i=1}^{2 N-1} W\left(\gamma_{i}\right)
$$

For $N=1$, the left-hand side misses the bond from 0 to len $(\gamma)$, so that in this case, the right-hand side has to be devided by $(1-\beta)$. Inserting this into the definition of $\Pi^{(N)}$ gives

$$
\begin{aligned}
& \Pi^{(1)}(x) \begin{cases}\leq \frac{\beta}{1-\beta} G^{\mathrm{saw}}(0) & \text { for } x=0, \\
=0 & \text { for } x \neq 0,\end{cases} \\
& \left|\Pi^{(2)}(x)\right| \leq \beta^{2} G^{\mathrm{saw}}(x)^{3},
\end{aligned}
$$

and, for $N \geq 2$,

$$
\left|\Pi^{(N)}(x)\right| \leq \beta^{N} \sum_{x_{2}, \ldots, x_{N-1}} G^{\mathrm{saw}}\left(x_{1}-x_{2}\right) \prod_{j=2}^{N}\left[G^{\mathrm{saw}}\left(x_{j}-x_{j-1}\right) G^{\mathrm{saw}}\left(x_{j+1}-x_{j-1}\right)\right],
$$

with $x_{1}=0, x_{N}=x_{N+1}=x$ (see again Figure 1).

To derive (20), observe that the terms $U_{s t}$ in (18) give the factor $\beta^{N}$ as well as restrictions $\gamma\left(a_{i}\right)=\gamma\left(b_{i}\right)$ for all $i$. Under this restrictions $\gamma$ breaks into paths $\gamma_{i}$ which are independent given their endpoints, so their sum gives $G^{\text {saw }}$. This implies (20).

With these estimates in hand, we can bound $\Pi$.

$\Pi^{(1)}$ clearly poses no problems. For $\Pi^{(2)}$ we have $\left|\Pi^{(2)}(x)\right| \leq \beta^{2} G^{\text {saw }}(x)^{3}$. By our assumptions $G^{\text {saw }}(x)^{3} \leq$ $27 G^{\mathrm{rw}}(x)^{3} \leq C|x|^{6-3 d}$, as required. For the terms with $N \geq 3$, we need the following lemma:

Lemma 3. Let $d>4$. For any $u, v \in \mathbb{Z}^{d}$,

$$
\sum_{w \in \mathbb{Z}^{d}}|w|^{4-2 d}|w-u|^{2-d}|w-v|^{2-d} \leq C|u|^{2-d}|v|^{2-d} .
$$

Proof. By Cauchy-Schwarz

$$
\left(\sum_{w \in \mathbb{Z}^{d}}|w|^{4-2 d}|w-u|^{2-d}|w-v|^{2-d}\right)^{2} \leq\left(\sum_{w \in \mathbb{Z}^{d}}|w|^{4-2 d}|w-u|^{4-2 d}\right)\left(\sum_{w \in \mathbb{Z}^{d}}|w|^{4-2 d}|w-v|^{4-2 d}\right)
$$

For $d>4$, each term can be estimated simply by splitting the sum to $|w|>|u| / 2$ and $|w| \leq|u| / 2$, see a detailed calculation in [9, Proposition 1.7(i)]. We get

$$
\sum_{w \in \mathbb{Z}^{d}}|w|^{4-2 d}|w-u|^{4-2 d} \leq C|u|^{4-2 d} .
$$

To use this lemma, define $A^{(N)}(x)$ by the right-hand side of (20) without the factor $\beta^{N}$, and $G(z)$ replaced by $|z|^{2-d}$. We prove by induction on $N \geq 2$ that

$$
A^{(N)}(x) \leq C^{N}|x|^{6-3 d} .
$$


As $A^{(2)}(x)=\left(|x|^{2-d}\right)^{3}$, the statement is true for $N=2$. Let $N>3$. In the expressions which are summed in $A^{(N)}$, we take out everything which depends on $x_{2}$ which is the factor

$$
\left|x_{2}\right|^{4-2 d}\left|x_{3}-x_{2}\right|^{2-d}\left|x_{4}-x_{2}\right|^{2-d} .
$$

The rest of the product is

$$
\left|x_{3}\right|^{2-d} \prod_{j=4}^{N}\left[\left|x_{j}-x_{j-1}\right|^{2-d}\left|x_{j+1}-x_{j-1}\right|^{2-d}\right] .
$$

For $N=3$, the product $\prod_{j=4}^{N}$ is absent and $x_{3}=x$. Summing over $x_{2}$ in (23) we can estimate this part with Lemma 3 by $C\left|x_{3}\right|^{2-d}\left|x_{4}\right|^{2-d}$ which together with (24) and summed over the other variables is $C A_{N-1}(x)$. (Remember that we had $x_{N+1}=x_{N}=x$.) This proves (22).

Using (22) together with $G^{\text {saw }}(x) \leq 3 G^{\mathrm{rw}}(x) \leq C|x|^{2-d}$ proves $\left|\Pi^{(N)}(x)\right| \leq(C \beta)^{N}|x|^{6-3 d}$ for $N \geq 2$. Together with (19), and summing over $N$, for sufficiently small $\beta$, this proves Lemma 1 .

\section{Proof of Lemma 2, with Banach algebras}

We start by defining a norm on $f: \mathbb{Z}^{d} \rightarrow \mathbb{R}$ by

$$
\|f\|=\max \left\{\sum_{x \in \mathbb{Z}^{d}}|f(x)|, \sup _{x \in \mathbb{Z}^{d}}|f(x)| \cdot|x|^{d}\right\} \in[0, \infty],
$$

where $|x|$ denotes, say, the $\ell^{2}$ norm in $\mathbb{Z}^{d}$. Our norm is a Banach algebra norm with respect to convolution, up to a constant. Indeed, let $f$ and $g$ satisfy that $\|f\|,\|g\|<\infty$. Then

$$
\sum_{x \in \mathbb{Z}^{d}}|(f * g)(x)| \leq\left(\sum_{x}|f(x)|\right)\left(\sum_{x}|g(x)|\right) \leq\|f\| \cdot\|g\|,
$$

and for every $x \in \mathbb{Z}^{d}$,

$$
|(f * g)(x)| \leq \sum_{y \in \mathbb{Z}^{d}}|f(y)||g(x-y)|=\sum_{|y|>|x-y|}|f(y)||g(x-y)|+\sum_{|y| \leq|x-y|}|f(y)||g(x-y)| .
$$

For the first term, whenever $|y|>|x-y|$, we have $|y|>|x| / 2$ and hence

$$
\sum_{|y|>|x-y|}|f(y)||g(x-y)| \leq \sup _{|y|>\frac{1}{2}|x|}|f(y)| \cdot \sum_{|y|>|x-y|}|g(x-y)| \leq\left(\frac{1}{2}|x|\right)^{-d}\|f\| \cdot\|g\| .
$$

A similar estimate holds for the other term, now using that $|x-y| \geq|x| / 2$ when $|y| \leq|x-y|$, and we get

$$
|(f * g)(x)| \leq 2^{d+1}|x|^{-d}\|f\|\|g\| .
$$

With (25) we get

$$
\|f * g\| \leq 2^{d+1}\|f\| \cdot\|g\| .
$$

In particular $B=\{f:\|f\|<\infty\}$ has a Banach algebra structure. In fact, if we define $\|f\|^{\prime}=2^{d+1}\|f\|$ then $B$ equipped with $\|\cdot\|^{\prime}$ is a Banach algebra (see also [11, Theorem VIII.1.4] for a proof that there exists an equivalent norm satisfying that the norm of the unit element is 1 ). But let us stick to using the norm $\|\cdot\|$. The only property of 
Banach algebras that we will use is that if $\left\|f-\delta_{0}\right\|<2^{-d-1}$, then $f$ is invertible. This follows because the inverse can be defined by the convergent Neumann series

$$
f^{-1}=\sum_{n=0}^{\infty}\left(\delta_{0}-f\right)^{n}
$$

In addition we get the norm estimate

$$
\left\|f^{-1}-\delta_{0}\right\| \leq \sum_{n=1}^{\infty}\left\|\left(\delta_{0}-f\right)^{n}\right\| \leq \sum_{n=1}^{\infty}\left(2^{d+1}\right)^{n-1}\left\|\delta_{0}-f\right\|^{n}=\frac{\left\|\delta_{0}-f\right\|}{1-2^{d+1}\left\|\delta_{0}-f\right\|} .
$$

We will not need anything else from the theory of Banach algebras.

The following lemma forms the heart of our analysis:

Lemma 4. Fix $d>2$. Assume $\rho: \mathbb{Z}^{d} \rightarrow \mathbb{R}$ satisfies

(i) $\rho$ is symmetric to coordinate permutations and flipping,

(ii) $\sum_{x} \rho(x)=0$

(iii) $|\rho(x)| \leq|x|^{-d-4}$.

Then $\left\|\rho * G^{\mathrm{rw}}\right\| \leq C$.

Proof. By [17], the random walk Green's function has an expansion of the form

$$
G^{\mathrm{rw}}(x)=a|x|^{2-d}+b|x|^{-d}+O\left(|x|^{-d-2}\right)
$$

(such an expansion is sometimes called an "Edgeworth expansion"). Therefore, using (iii) and (27) (in fact here the simpler (2) is enough), the sum defining $\left(\rho * G^{\mathrm{rw}}\right)(x)$ converges absolutely for every $x \in \mathbb{Z}^{d}$ so this function is well defined. Write

$$
\left(\rho * G^{\mathrm{rw}}\right)(x)=\sum_{y} \rho(y) G^{\mathrm{rw}}(x-y)=\sum_{|y|<\frac{1}{2}|x|} \rho(y) G^{\mathrm{rw}}(x-y)+\sum_{|y| \geq \frac{1}{2}|x|} \rho(y) G^{\mathrm{rw}}(x-y)=I+I I .
$$

We start with $I$ and write it as $I=I_{1}+I_{2}+I_{3}, I_{j}$ from the three parts on the RHS of (27). For $I_{1}$ we Taylor expand $|x-y|^{2-d}$ around $x$ to order 3 and get

$$
\begin{aligned}
\frac{1}{a} I_{1}= & \sum_{|y|<\frac{1}{2}|x|} \rho(y)|x-y|^{2-d}=\sum_{|y|<\frac{1}{2}|x|} \rho(y)\left[|x|^{2-d}+\sum_{i=1}^{d} y_{i} \cdot(2-d) x_{i}|x|^{-d}\right. \\
& \left.+\sum_{i, j=1}^{d} y_{i} y_{j}\left(-(2-d) d \cdot x_{i} x_{j}|x|^{-d-2}+\delta_{i j}(2-d)|x|^{-d}\right)+O\left(|y|^{3}|x|^{-d-1}\right)\right] .
\end{aligned}
$$

We now bound these terms. For the first we write

$$
\left.\left.\left|\sum_{|y|<\frac{1}{2}|x|} \rho(y)\right| x\right|^{2-d}|=| x\right|^{2-d}\left|\sum_{|y| \geq \frac{1}{2}|x|} \rho(y)\right| \leq|x|^{2-d} \sum_{|y| \geq \frac{1}{2}|x|}|y|^{-d-4} \leq C|x|^{-d-2},
$$

where in the equality we have used that $\sum_{x} \rho(x)=0$. For the second, we use the symmetry of $\rho$ to flipping of $y_{i}$ to conclude that

$$
\sum_{|y|<\frac{1}{2}|x|} \rho(y) y_{i}=0
$$


and similarly for the off-diagonal second-order terms, i.e., for $\sum_{|y|<\frac{1}{2}|x|} \rho(y) y_{i} y_{j}$ for $i \neq j$. The on-diagonal terms are equal to

$$
\sum_{i=1}^{d} \sum_{|y|<\frac{1}{2}|x|} \rho(y) y_{i}^{2}\left((2-d)|x|^{-d}-(2-d) d \cdot x_{i}^{2}|x|^{-d-2}\right),
$$

and the symmetry of $\rho$ to coordinate permutations shows that $\sum_{|y|<\frac{1}{2}|x|} \rho(y) y_{i}^{2}$ does not depend on $i$. We take it out of the sum and see that

$$
\sum_{i=1}^{d}\left((2-d)|x|^{-d}-(2-d) d \cdot x_{i}^{2}|x|^{-d-2}\right)=0 .
$$

Finally, the third order terms are bounded by

$$
\sum_{|y|<\frac{1}{2}|x|}|\rho(y)||y|^{3}|x|^{-d-1} \leq|x|^{-d-1} \sum_{|y|<\frac{1}{2}|x|}|y|^{-d-4}|y|^{3} \leq C|x|^{-d-1} .
$$

Putting all these estimates together gives that

$$
\left|I_{1}\right| \leq C|x|^{-d-1} \text {. }
$$

The estimates of $I_{2}, I_{3}$ and $I I$ are much simpler. To estimate $\left|I_{2}\right|$, we Taylor expand $|x-y|^{-d}$ to first order, i.e. $|x-y|^{-d}=|x|^{-d}+O\left(|y||x|^{-d-1}\right.$ ). A similar argument shows that $\left|I_{2}\right| \leq C|x|^{-d-1}$. (We don't need the harmonicity of $|x|^{2-d}$ which is the true reason for the cancellation in (28) above.) For $I_{3}$, we bound, again using that $|x-y|>\frac{1}{2}|x|$ when $|y|<\frac{1}{2}|x|$,

$$
\left|I_{3}\right| \leq C \sum_{|y|<\frac{1}{2}|x|}|\rho(y)||x-y|^{-d-2} \leq C|x|^{-d-2} \sum_{y \in \mathbb{Z}^{d}}|\rho(x)| \leq C|x|^{-d-2} .
$$

For $I I$, we split $I I=I I_{1}+I I_{2}$, depending on whether $|x-y| \leq|x|$ or $|x-y|>|x|$ and use $|y| \geq \frac{1}{2}|x|,|\rho(y)| \leq|y|^{-d-4}$ together with $G^{\mathrm{rw}}(x) \leq C|x|^{2-d}$ to bound

$$
\left|I I_{1}\right| \leq 2^{d+4}|x|^{-d-4} \sum_{y:|x-y| \leq|x|} G^{\mathrm{rw}}(x-y) \leq C|x|^{-d-2},
$$

while for $|x-y|>|x|$,

$$
\left|I_{2}\right| \leq C|x|^{2-d} \sum_{|y| \geq \frac{1}{2}|x|}|\rho(y)| \leq C|x|^{2-d}|x|^{-4}=C|x|^{-d-2} .
$$

We conclude that $\left|\left(\rho * G^{\mathrm{rw}}\right)(x)\right| \leq C|x|^{-d-1}$. This proves the lemma.

Remark. It seems as if Lemma 4 makes a stringent requirement on the types of random walks for which the argument can be applied, as Edgeworth expansions are not easy to get. For example, if one wishes to apply the argument for the Cayley graph of, say, the Heisenberg group, then the natural analog of an Edgeworth expansion is not known. We have a more roundabout proof of Lemma 4 that only uses the local central limit theorem. This argument will be presented elsewhere.

In the following lemma, we extend Lemma 4 to the subcritical SRW Green's function $G_{\mu}^{\mathrm{rw}}$, i.e., $G_{\mu}^{\mathrm{rw}}(x)=$ $\sum_{n \geq 0}(2 d \mu)^{n} p_{n}(x)$. Note that $G_{\mu}^{\mathrm{rw}} * \Delta_{\mu}^{\mathrm{rw}}=\delta_{0}$. 
Lemma 5. Let $d>2$. Let $\rho$ be as in Lemma 4 and let $\mu \in\left[-\frac{1}{4 d}, \frac{1}{2 d}\right]$. Then

$$
\left\|\rho * G_{\mu}^{\mathrm{rw}}\right\| \leq C
$$

where $C$ does not depend on $\mu$.

Proof. Write

$$
\rho * G_{\mu}^{\mathrm{rw}}=\rho * G^{\mathrm{rw}} * \Delta^{\mathrm{rw}} * G_{\mu}^{\mathrm{rw}}
$$

(recall that $G^{\mathrm{rw}}=G_{1 /(2 d)}^{\mathrm{rw}}$ and $\left.\Delta^{\mathrm{rw}}=\Delta_{1 /(2 d)}^{\mathrm{rw}}\right)$. Notice that we do not need to put any parenthesis in this expression as associativity follows from the fact that all sums converge absolutely, which can be easily seen from the upper bounds for the various terms. Since we already know that $\left\|\rho * G^{\mathrm{rw}}\right\| \leq C$ by Lemma 4 , we need only bound $\left\|G_{\mu}^{\mathrm{rw}} * \Delta^{\mathrm{rw}}\right\|$ (note again that this is not $\Delta_{\mu}^{\mathrm{rw}}$ but rather $\left.\Delta_{1 /(2 d)}^{\mathrm{rW}}\right)$. Noting that $p_{n} * p_{m}=p_{n+m}$, we get

$$
G_{\mu}^{\mathrm{rw}} * \Delta^{\mathrm{rw}}=\left(\sum_{n=0}^{\infty}(2 d \mu)^{n} p_{n}\right) *\left(\delta_{0}-p_{1}\right)=\delta_{0}-(1-2 d \mu) \sum_{n=1}^{\infty}(2 d \mu)^{n-1} p_{n} .
$$

For $\mu=1 /(2 d)$, this is identically equal to $\delta_{0}$, and there is nothing to prove. Thus, we can assume that $\mu \in\left[-\frac{1}{4 d}, \frac{1}{2 d}\right)$. Now, since $p_{n}(x) \leq C n^{-d / 2} \mathrm{e}^{-c|x|^{2} / n}$ we get that $\left\|p_{n}\right\| \leq C$. Hence,

$$
\left\|G_{\mu}^{\mathrm{rw}} * \Delta^{\mathrm{rw}}\right\| \leq 1+(1-2 d \mu) \sum_{n=1}^{\infty}(2 d \mu)^{n-1}\left\|p_{n}\right\| \leq C,
$$

and the lemma is proved.

Proof of Lemma 2. Recall that the input of the lemma is a function $\Delta$ satisfying conditions (i)-(iii) of Lemma 1. These conditions are quite close to the conditions on $\rho$ in Lemmas 4 and 5, only a linear map is required to pass from one to the other. We define $\mu$ by

$$
\mu=\frac{1}{2 d}\left(1-\sum_{x \in \mathbb{Z}^{d}} \Delta(x)\right)
$$

which implies that

$$
\sum_{x \in \mathbb{Z}^{d}}\left(\Delta-\Delta_{\mu}^{\mathrm{rw}}\right)(x)=0
$$

(This choice is closely related to the choice of constants $\lambda, \mu$ in $[9,(2.29)]$.) To use Lemma 5 we need to justify $\mu \in\left[-\frac{1}{4 d}, \frac{1}{2 d}\right]$. The upper bound $\mu \leq \frac{1}{2 d}$ is automatic since $\sum_{x} \Delta(x) \geq 0$. For the lower bound $\mu \geq-\frac{1}{4 d}$, we need to show that $\sum_{x} \Delta(x) \leq \frac{3}{2}$. This follows for small enough $\beta$ by our assumption that $\Delta$ satisfies (iii) of Lemma 1 and $\sum_{x} \Delta_{\lambda^{\prime}}^{\mathrm{rw}}(x) \leq 1$.

Next we note that

$$
\left|\Delta(x)-\Delta_{\mu}^{\mathrm{rw}}(x)\right| \leq C_{2} \beta|x|^{-d-4} .
$$

Indeed, at every $x$ that is not a neighbour of 0 this is an immediate corollary from our condition (iii) of Lemma 1. For the neighbours, we note that

$$
\left|\lambda^{\prime}-\mu\right|=\frac{1}{2 d}\left|\sum_{x \in \mathbb{Z}^{d}}\left(\Delta_{\lambda^{\prime}}^{\mathrm{rw}}-\Delta_{\mu}^{\mathrm{rw}}\right)(x)\right| \stackrel{(29)}{=} \frac{1}{2 d}\left|\sum_{x \in \mathbb{Z}^{d}}\left(\Delta_{\lambda^{\prime}}^{\mathrm{rw}}-\Delta\right)(x)\right| \leq \frac{C \beta}{2 d},
$$


where the last inequality is again from condition (iii) of Lemma 1. Thus, for $x$ a neighbour of the origin, we conclude that

$$
\Delta(x)-\Delta_{\mu}^{\mathrm{rw}}(x)=\mu-\lambda^{\prime}+\Delta(x)-\Delta_{\lambda^{\prime}}^{\mathrm{rw}}(x)=O(\beta),
$$

by (31) and condition (iii) of Lemma 1 . This shows (30).

We next define

$$
\rho=\frac{1}{C_{2} \beta}\left(\Delta-\Delta_{\mu}^{\mathrm{rw}}\right),
$$

with $C_{2}$ being the constant from (30). This $\rho$ has the properties (i)-(iii) of Lemma 4, so that

$$
\left\|\rho * G_{\mu}^{\mathrm{rw}}\right\| \leq C .
$$

In turn, this implies

$$
\left\|\left(\Delta-\Delta_{\mu}^{\mathrm{rw}}\right) * G_{\mu}^{\mathrm{rw}}\right\| \leq C \beta .
$$

But this is exactly $\Delta * G_{\mu}^{\mathrm{rw}}-\delta_{0}$. This means that $\Delta * G_{\mu}^{\mathrm{rw}}$ is invertible if $\beta$ is sufficiently small (recall (26)), and further that we have $\left(\Delta * G_{\mu}^{\mathrm{rw}}\right)^{-1}=\delta_{0}+E$ with $\|E\| \leq C \beta$. Our required function is now

$$
G=\left(\Delta * G_{\mu}^{\mathrm{rw}}\right)^{-1} * G_{\mu}^{\mathrm{rw}},
$$

which is clearly an inverse for $\Delta$. To see that $G(x) \leq 2 G^{\mathrm{rw}}(x)$ write

$$
G=G_{\mu}^{\mathrm{rw}}+E * G_{\mu}^{\mathrm{rw}} .
$$

Since $G_{\mu}^{\mathrm{rw}}(x) \leq G^{\mathrm{rw}}(x)$, because $\mu \leq 1 /(2 d)$, we need only estimate $E * G_{\mu}^{\mathrm{rw}}$. We write

$$
\left(E * G_{\mu}^{\mathrm{rw}}\right)(x)=\sum_{y} G_{\mu}^{\mathrm{rw}}(y) E(x-y)=\sum_{|y|<\frac{1}{2}|x|} G_{\mu}^{\mathrm{rw}}(y) E(x-y)+\sum_{|y| \geq \frac{1}{2}|x|} G_{\mu}^{\mathrm{rw}}(y) E(x-y)=I+I I .
$$

For $I$, we use that $|y|<\frac{1}{2}|x|$ implies that $|x-y| \geq \frac{1}{2}|x|$ so $|E(x-y)| \leq C \beta|x|^{-d}$ and hence

$$
|I| \leq C \beta|x|^{-d} \sum_{|y|<\frac{1}{2}|x|}\left|G_{\mu}^{\mathrm{rw}}(y)\right| \leq C \beta|x|^{-d} \sum_{|y|<\frac{1}{2}|x|}|y|^{2-d} \leq C \beta|x|^{2-d} .
$$

For $I I$, we have

$$
|I I| \leq\left(\max _{|y| \geq \frac{1}{2}|x|}\left|G_{\mu}^{\mathrm{rw}}(y)\right|\right) \cdot \sum_{y}|E(x-y)| \leq C\left(\frac{1}{2}|x|\right)^{2-d} \cdot C \beta=C \beta|x|^{2-d} .
$$

We get that $\left|\left(E * G_{\mu}^{\mathrm{rw}}\right)\right| \leq C \beta|x|^{2-d}$, which means that for $\beta$ sufficiently small, it is less than $G^{\mathrm{rw}}$. This shows that $G(x) \leq 2 G^{\mathrm{rw}}(x)$, and thus completes the proof of Lemma 2 .

\section{Remarks}

(1) Examining (32)-(35) in the proof of the last lemma shows that in fact we got that

$$
G_{\lambda}^{\mathrm{saw}}(x)=G_{\mu}^{\mathrm{rw}}(x)+O\left(\beta|x|^{2-d}\right) .
$$

Together with (27), this would prove (3), if only we could show that $\mu=\frac{1}{2 d}$ for $\lambda_{c}$, the critical $\lambda$. This is a classical fact, let us sketch its proof for the convenience of the reader. Since $\mu=\frac{1}{2 d}\left(1-\sum_{x} \Delta(x)\right)$, it is equivalent to showing 
that $\chi(\lambda) \rightarrow \infty$ as $\lambda \nearrow \lambda_{c}$ where $\chi(\lambda)=\sum_{x} G_{\lambda}^{\text {saw }}(x)$ (recall (14)). Let $c_{n}=\sum_{\operatorname{len}(\gamma)=n} W^{\beta}(\gamma)$ so $\chi(\lambda)=\sum_{n} \lambda^{n} c_{n}$. Then, $c_{n}$ is submultiplicative i.e. $c_{n} \leq c_{m} c_{n-m}$, so $c_{n} \geq \lambda_{c}^{n}$. Summing gives $\chi(\lambda) \geq 1 /\left(\lambda_{c}-\lambda\right)$. (The reader who finds this sketch too dense may see more details in, say, [16, Theorem 2.3].)

(2) The result of the theorem is known as an "infrared bound." It implies the finiteness of the so-called bubble diagram, which in turn implies various critical exponents. See again [16, Theorem 2.3].

(3) Let us remark on the exponent $-d-4$ appearing in the inequality $\left|\Delta^{\mathrm{rw}}-\Delta^{\mathrm{saw}}\right| \leq C \beta|x|^{-d-4}$ of Lemma 1 . We in fact prove a stronger bound with exponent $-3(2-d)$, recall (12). Most of the proof of Lemma 2 actually needs less, $|x|^{-d-2-\varepsilon}$ would have been enough. The only place where the stronger estimate $|x|^{-d-4}$ is used is in Lemma 5, in order to justify the associativity of the convolution in the expression $\rho * G^{\mathrm{rw}} * \Delta^{\mathrm{rw}} * G_{\mu}^{\mathrm{rw}}$. There are certainly ways to justify associativity at that point under the weaker assumption $|\rho(x)| \leq|x|^{-d-2-\varepsilon}$, but an additional argument would be needed.

\section{Acknowledgements}

This work was performed in part during a visit of RvdH to the Weizmann Institute, and when the authors met in Eurandom, Oberwolfach and the Institut Henri Poincaré. We thank these institutions for their hospitality.

\section{References}

[1] L. Avena, E. Bolthausen and C. Ritzmann. A local CLT for convolution equations with an application to weakly self-avoiding random walks. Ann. Probab. 44 (1) (2016) 206-234. MR3456336

[2] E. Bolthausen and C. Ritzmann. A central limit theorem for convolution equations and weakly self-avoiding walks. Unpublished manuscript, 2001. Available at arXiv:math/0103218.

[3] C. Borgs, J. T. Chayes, R. van der Hofstad, G. Slade and J. Spencer. Random subgraphs of finite graphs. II. The lace expansion and the triangle condition. Ann. Probab. 33 (5) (2005) 1886-1944. MR2165583

[4] D. Brydges and T. Spencer. Self-avoiding walk in 5 or more dimensions. Comm. Math. Phys. 97 (1-2) (1985) 125-148. MR0782962

[5] L.-C. Chen and A. Sakai. Critical two-point functions for long-range statistical-mechanical models in high dimensions. Ann. Probab. 43 (2) (2015) 639-681. MR3306002

[6] T. Hara. Decay of correlations in nearest-neighbor self-avoiding walk, percolation, lattice trees and animals. Ann. Probab. 36 (2) (2008) 530-593. MR2393990

[7] T. Hara and G. Slade. Mean-field critical behaviour for percolation in high dimensions. Comm. Math. Phys. 128 (2) (1990) $333-391$. MR1043524

[8] T. Hara and G. Slade. On the upper critical dimension of lattice trees and lattice animals. J. Stat. Phys. 59 (5-6) (1990) $1469-1510$. MR1063208

[9] T. Hara, R. van der Hofstad and G. Slade. Critical two-point functions and the lace expansion for spread-out high-dimensional percolation and related models. Ann. Probab. 31 (2) (2003) 349-408. MR1959796

[10] R. van der Hofstad, F. den Hollander and G. Slade. A new inductive approach to the lace expansion for self-avoiding walks. Probab. Theory Related Fields 111 (2) (1990) 253-286. MR1633582

[11] Y. Katznelson. An Introduction to Harmonic Analysis, 3rd edition. Cambridge Mathematical Library. Cambridge University Press, Cambridge, 2004. MR2039503

[12] A. Sakai. Mean-field critical behavior for the contact process. J. Stat. Phys. 104 (1-2) (2001) 111-143. MR1851386

[13] A. Sakai. Lace expansion for the Ising model. Comm. Math. Phys. 272 (2) (2007) 283-344. MR2300246

[14] A. Sakai. Application of the lace expansion to the $\varphi^{4}$ model. Comm. Math. Phys. 336 (2) (2015) 619-648. MR3322382

[15] G. Slade. The diffusion of self-avoiding random walk in high dimensions. Comm. Math. Phys. 110 (4) (1987) 661-683. MR0895223

[16] G. Slade. The lace expansion and its applications. In Lectures from the 34th Summer School on Probability Theory Held in Saint-Flour, July 6-24. J. Picard (Ed). Lecture Notes in Mathematics 1879. Springer-Verlag, Berlin, 2004. MR2239599

[17] K. Uchiyama. Green's functions for random walks on $\mathbb{Z}^{N}$. Proc. Lond. Math. Soc. (3) 77 (1) (1998) 215-240. MR1625467 